OP0052 CORTICAL BONE LOSS IS AN EARLY FEATURE OF AXIAL SPONDYLOARTHRITIS

J. Haschka ${ }^{1,2}$, A. Neumann ${ }^{1}$, A. Kleyer ${ }^{1}$, L. Schuster ${ }^{1}$, M. Englbrecht ${ }^{1}$, C.P. Figueiredo ${ }^{1,3}$, C. Muschitz ${ }^{2}$, R. Kocijan ${ }^{2}$, H. Resch ${ }^{2}$, J. Rech ${ }^{1}$, G. Schett ${ }^{1}$. ${ }^{1}$ Department of Internal Medicine 3, University of Erlangen-Nuremberg, Erlangen, Germany; ${ }^{2}$ Department of Internal Medicine II, St. Vincent Hospital, VINFORCE Study Group, Vienna, Austria; ${ }^{3}$ Division of Rheumatology, Faculdade de Medicina da Universidade de São Paulo, São Paulo, Brazil

Background: Systemic bone loss is a well-known and severe consequence in axial spondyloarthritis (axSpA). To date deterioration of bone microstructure has only been described in patients with long-standing ankylosing spondylitis while bone microstructure has not been assessed in axSpA.

Objectives: The aim of the present study was to investigate bone microstructure, geometry and volumetric bone mineral density (VBMD) using high resolution peripheral quantitative CT (HR-pQCT) in a cohort of axSpA patients at an early stage of disease and to search for potential factors for deterioration of bone microstructure.

Methods: An inception cohort of 101 axSpA patients and 50 healthy controls of similar age and sex was assessed for geometric, volumetric and microstructural parameters of bone using HR-pQCT scanning of the radius. Additionally, demographic and disease specific characteristics of SpA patients were recorded. Results: SpA patients and controls were comparable in age (median (IQR) 45.0 (15.0) vs. 44.76 (26.0) years, $p=0.917$ ), sex (female $41.6 \%$ vs. $40 \%, p=0.852$ ) and BMI (median (IQR) 26.3 (6.5) vs. 23.8 (5.2), $\mathrm{p}=0.118$ ). $75 \%$ of patients showed HLA-B27 positivity. Median disease duration was $6.5(9.0)$ years, $58.4 \%$ of patients were on biological treatment and $14.9 \%$ of patients in disease remission according to ASDAS-CRP. Geometric and microstructural analysis by HR-pQCT revealed a significantly reduced cortical area $(p=0.022)$ and cortical thickness $(p=0.006)$ in $\mathrm{SpA}$ patients compared to controls. No differences in cortical porosity $(p=0.685)$, trabecular geometry or microstructure were detected. Total and cortical vBMD were significantly reduced in SpA patients $(p=0.042$ and $p=0.007)$, while there was no difference in trabecular vBMD $(p=0.376)$. Patients with a short disease duration $<2$ years $(n=46)$ showed a significant reduction of cortical thickness and cortical area $(\mathrm{p}=0.050$ and $\mathrm{p}=0.032)$ compared to controls. Patients with a disease duration $>2$ years $(n=55)$ additionally developed a decrease of cortical and total $\operatorname{vBMD}(p=0.004$ and $p=0.036)$. Multivariate regression models identified male sex to be associated with lower cortical VBMD and female sex with lower trabecular vBMD. History of prednisolone treatment $(>5 \mathrm{mg}>3$ months) was associated with lower trabecular VBMD, and disease duration with higher trabecular VBMD. Remission status, treatment with TNF inhibitors, HLA-B27 status and presence of peripheral arthritis did not influence bone microstructure independently.

Conclusions: Bone microstructure in SpA patients is primarily characterized by deterioration of cortical bone. Cortical bone loss starts early and is evident within the first 2 years of disease.

Disclosure of Interest: None declared

DOI: 10.1136/annrheumdis-2017-eular.3888

\section{OP0053 BONE LOSS AND CARDIOVASCULAR RISK IN PATIENTS WITH EROSIVE AND NON-EROSIVE HAND OSTEOARTHRITIS}

O. Ruzickova, O. Sleglova, K. Pavelka, L. Senolt. Revmatology, Institute of revmatology, Prague 2, Czech Republic

Background: Hand osteoarthritis $(\mathrm{OA})$ and its more severe subset erosive hand $\mathrm{OA}$ are common causes of pain and morbidity. Some metabolic factors were suggested to be implicated in erosive disease. Furthermore, few studies investigated differences in systemic bone loss and cardiovascular risk factors between erosive and non-erosive hand OA.

Objectives: To compare bone mineral density (BMD) and major cardiovascular risk factors between patients with erosive and non-erosive hand $\mathrm{OA}$ in a cross-sectional study.

Methods: Patients with symptomatic disease fulfilling the American College of Rheumatology (ACR) criteria for hand OA were included in this study. Erosive hand OA was defined by at least one erosive interphalangeal joint. All patients underwent clinical assessments of joint swelling and radiographs of both hands. DEXA examination of lumbar spine, total femur and femur neck was performed. Metabolic risk factors (body mass index, hypertension, diabetes, dyslipidaemia) were collected. Patients were examined at baseline, one-year and two years follow-up. Results: Altogether, 129 patients (12 male) with symptomatic nodal hand OA were included in this study and followed between April 2012 and January 2017. Out of these patients, 72 had erosive disease. The disease duration $(p<0.01)$ was significantly higher in patients with erosive compared with non-erosive disease at baseline. Patients were taking symptomatic slow acting drugs (SYSADOA) twice a year, non-steroidal anti-inflammatory drugs (NSAIDs) and analgesics on demand. Baseline population characteristics did not differ between both groups. Osteoporosis (T-score <-2.5 SD) was diagnosed in 12.5\% (9/72) of patients with erosive hand $\mathrm{OA}$ and in $8.06 \%(5 / 57)$ of patients with non-erosive hand $\mathrm{OA}$. Although bone mineral density did not significantly differ between the groups, T-scores of lumbar spine $(-0.46$ vs. $-1.04 S D, p<0.001)$, total femur $(-0.36$ vs. -1.20 SD, $p<0.001)$ and femur neck $(-0.92$ vs. -1.20 SD, $p<0.01$ ) were significantly lower in patients with erosive compared with non-erosive disease. After two years, the decrease in T- score of lumbar spine was significantly higher in patients with erosive compared with non-erosive hand $\mathrm{OA}(-0.08 \mathrm{SD}$ vs. $0.07 \mathrm{SD}, \mathrm{p}<0.01$; total difference between groups is $10.92 \%$ ). The decrease of T-score in femur neck, total femur and the decrease of BMD $\left(\mathrm{g} / \mathrm{cm}^{2}\right)$ in all regions were also higher, although not significantly, in patients with erosive compared with non-erosive hand OA. In addition, more patients with erosive compared with non-erosive hand OA were treated for dyslipidaemia at baseline and after two years (32\% vs. $28 \%$ and $32 \%$ vs. $30 \%, p<0.01$ for both comparisons).

Conclusions: These results suggest that patients with erosive hand $O A$ are at risk for development of general bone loss and cardiovascular diseases.

Acknowledgements: This work was supported by the project MHCR No. 023728. Disclosure of Interest: None declared

DOI: 10.1136/annrheumdis-2017-eular.6064

\section{OP0054 INCIDENCE OF VERTEBRAL FRACTURES IN EARLY SPONDYLOARTHRITIS: 5-YEAR PROSPECTIVE DATA OF THE DESIR COHORT}

J. Sahuguet $^{1}$, J. Fechtenbaum ${ }^{1}$, A. Molto ${ }^{1}$, A. Etcheto ${ }^{1}$, P. Richette ${ }^{2}$, M. Dougados ${ }^{1}$, C. Roux ${ }^{1}$, K. Briot ${ }^{1} .{ }^{1}$ Rheumatology department, Hôpital Cochin; ${ }^{2}$ Rheumatology department, Hôpital Lariboisière, Paris, France

Background: Osteoporosis is a well-recognized feature of axial spondyloartyhritis (ax-SpA) and an increased risk of vertebral fractures (VFs) has been reported in patients with SpA. The prevalence of VFs is highly variable across studies, up to $30 \%$. These data are unexpected in a disease affecting a young population, predominantly males. These results could be related to differences in methods of diagnoses of VFs and in populations with highly variable duration of the disease. We hypothesized that the prevalence and incidence of VFs be lower than the ones previously reported, especially in early spondyloarthritis.

Objectives: Our primary aim was to assess the prevalence of VFs in a cohort of early inflammatory back pain suggestive of early axial SpA and their incidence over 5 years.

Methods: Study population: patients from the DESIR (DEvenir des Spondylarthropathies Indifférenciées Récentes) cohort, which is a french national longitudinal prospective cohort including adults between 18 and 50 years old, and presenting with inflammatory back pain suggestive of axSpA for less than 3 years. Follow-up is still ongoing, but the data presented here includes the first 5 years of follow-up. All patients had thoracic and lumbar spine X-rays at baseline, 2 years and 5 years. For this particular study, all radiographs of the DESIR cohort were centrally read by one reader, an expert in the field of the diagnosis of VFs according to Genant's method. Careful assessment was used to distinguish true VFs not to be mistaken with deformities. Using a temporal sequence of reading (i.e. unblinded for chronological order), an incident VF was defined as a change in the score of a vertebra from grade 0 to a subsequent grade 1 or more. All vertebrae between T4 and L4 were evaluated. In doubtful cases, an adjudication by two other senior experts was performed. Prevalence at inclusion and the incidence of VFs over the first 5 years of follow-up were described.

Results: A total of 708 patients with inflammatory back pain were included in the DESIR cohort. Plain dorsolumbar spine X-rays were available for 694 patients, and thoracic and/or lumbar X-rays were available for 643 patients at baseline. Twenty eight VFs were identified in 21 patients (19 grade 1 VFs and 9 Grade 2 or $3 \mathrm{VFs}$ ); therefore, the prevalence of VFs was $4.5 \%$.

Complete X-ray follow up between baseline and M60 was available for 433 patients. Seven incident VFs were identified in 6 patients: at 2 years 3 grade 1 VFs and 1 grade 2 VF were identified; and at 5 years, 2 grade 1 VF and 1 grade 2 or more VF were identified. The 5 -year incidence of VFs was $1.6 \%$.

Conclusions: In this study focused on a population of early spondyloarthritis, the reported low prevalence of VFs of $4.5 \%$ and incidence of $1.6 \%$ confirm our hypothesis that the real prevalence and incidence of VF in SpA is probably much lower than what was reported in previous studies. These discrepancies might be explained by the variability in the methods of vertebral fracture's assessment as vertebral deformations might be inappropriately considered as fracture.

Disclosure of Interest: None declared

DOI: 10.1136/annrheumdis-2017-eular.6324

WEDNESDAY, 14 JUNE 2017

Rehabilitation and modern drug treatment - needs and challenges

\section{OP0055-HPR PREFERENCES FOR SELF-MANAGEMENT AND SUPPORT SERVICES IN PATIENTS WITH INFLAMMATORY ARTHRITIS: A DANISH NATIONWIDE CROSS-SECTIONAL STUDY}

N.M. Hammer ${ }^{1}$, C. Flurey ${ }^{2}$, K.V. Jensen ${ }^{1}$, L. Andersen ${ }^{1}$, B.A. Esbensen ${ }^{1,3}$. ${ }^{1}$ Rigshospitalet - Glostrup, COPECARE, Centre for Rheumatology and Spine Diseases \& Research Unit, Glostrup, Denmark; ${ }^{2}$ Rheumatology Unit, Bristol Royal Infirmary, Bristol, West England, United Kingdom; ${ }^{3}$ Department of Clinical Medicine, Faculty of Health and Medical, University of Copenhagen, Copenhagen, Denmark

Background: During recent years the medical treatment of inflammatory arthritis 
(IA) has improved and become more efficient. However, many patients still continue to experience serious negative impact on both their physical and psychosocial health and wellbeing. Therefore, it is essential to identify patients' needs for support for managing IA in everyday life.

Objectives: To explore preferences for self-management and support services in patients with inflammatory arthritis.

Methods: Adult patients with IA (rheumatoid arthritis (RA), psoriatic arthritis (PsA) and axial spondyloarthritis (axSpA)) were invited through The Danish Rheumatism Organization, arthritis networks, and hospitals' rheumatology departments across the country to participate in a cross-sectional study using online survey methodology. The questionnaire included questions on patient's interest in participation (requiring an answer of yes or no) in a total of 30 different self-management and support services within eight overall categories (i.e. discussion groups, one-to-one sessions, question and answer sessions, organised talks, physical activity, education sessions, raising arthritis awareness events, and online services), and preferences regarding practical issues of taking part. Descriptive statistics were applied.

Results: In total, 664 patients (85\% female) responded, of which $53 \%$ had RA, $27 \%$ had PsA and $20 \%$ had axSpA. Respondents' mean age was 50 years $(S D=13)$, and median disease duration was 10 years (IQR=4-16). Of the 30 different self-management and support services, the most popular was Online service: Website for information (about symptoms, treatment and selfmanagement of arthritis) with $91 \%$ of the respondents indicating interest. This was followed by One-to-one session with a rheumatologist (about coping with arthritis) (89\%) and Organised talks by researchers (about current rheumatology research) (83\%). Also, One-to-one session with a nurse, One-to-one session with a physiotherapist, and Education session on managing symptoms were all chosen by more than $80 \%$ of the respondents. The vast majority of respondents $(81 \%)$ indicated to prefer a group with no fixed commitments and an advertised time table. Regarding timing of support, the majority $(70 \%)$ indicated that selfmanagement and support services should optimally be offered whenever needed. However, respondents also stated it would be helpful within the first six months of being diagnosed $(49 \%)$ as well as during flares $(30 \%)$.

Conclusions: Patients with IA show high overall interest in taking part in self-management and support services. Especially, websites for finding disease related information, one-to-one sessions with health professionals, organised talks about rheumatology research and education on symptom management are requested. The preference regarding practical issues seems to be for a flexible delivery according to the patients' fluctuating needs during their illness course. Disclosure of Interest: None declared

DOI: 10.1136/annrheumdis-2017-eular.5002

\section{WEDNESDAY, 14 JUNE 2017 \\ Basic and clinical science in paediatric rheumatology}

\section{OP0056 IL-23P19 IS UP-REGULATED IN MONOCYTE-DERIVED MACROPHAGES FROM HLA B27 POSITIVE PATIENTS WITH ENTHESITIS RELATED ARTHRITIS}

C. Fisher, D. Eleftheriou, D. Sen, Y. Ioannou. Arthritis Research UK Centre for Adolescent Rheumatology, University College London, London, United Kingdom

Background: Enthesitis related arthritis (ERA) is an HLAB27-associated subtype of JIA most similar to the adult spondyloarthropathies (SpA). The innate immune system, intracellular stress response (the unfolded protein response (UPR)) and IL23/IL17 pathway are strongly implicated in the pathogenesis of adult SpA. However, these systems remain relatively unexplored in ERA.

Objectives: To compare levels of the IL23 subunit, IL23p19, produced by monocyte-derived macrophages (MDMs) in the presence of lipopolysaccharide (LPS) and an inducer of the UPR (tunicamycin (TM)) from patients with ERA and healthy controls. Other pro-inflammatory cytokines and markers of the UPR were also studied.

Methods: Peripheral blood monocytes isolated from 30 patients with ERA (20 HLAB27 positive, 10 HLAB27 negative, median age 16yrs 4mths, median disease duration 3yrs 9mths, M:F 7:1) and 16 age and gender-matched healthy controls were differentiated in vitro with macrophage-colony stimulating factor. Cells were treated with IFN $\gamma$ and then stimulated with LPS alone or LPS with TM for 24 hours. Pro-inflammatory cytokines and markers of the UPR were measured by expression of mRNA using qPCR and normalised against GAPDH.
Results: IL23p19 expression was higher in MDMs from HLAB27 positive patients with ERA compared to healthy controls treated with LPS [median relative expression 384.7 (IQR 179.2-1340) vs 90.5 (49.9-455.9), $\mathrm{p}=0.02$ ]. With the addition of the UPR inducer, TM, enhanced IL23p19 mRNA expression was also seen in HLAB27 positive patients compared to those who were HLAB27 negative and healthy controls [median relative expression 608.9 (IQR 282-3286) vs 283.0 (IQR 20.1-928.2) vs 195.1 (IQR 9.8-853.7); $\mathrm{p}=0.02$ ]. When the groups were divided in to males and females, significantly higher IL23p19 expression was seen in MDMs treated with LPS from male HLAB27 positive patients with ERA compared to male healthy controls [661.7 (IQR 169.7-1531) vs 78.3 (39.01139.6), $p=0.0095]$ (figure 1). This was also the case for MDMs treated with LPS and TM [537.5 (IQR 295.3-3054) vs 93.05 (IQR 2.7-1109); $p=0.02]$. To investigate the effect of UPR induction on IL23p19 mRNA expression, percentage increase was calculated for each patient between MDMs treated with LPS alone and MDMs treated with LPS and TM. Interestingly, median percentage increase for HLAB27 positive patients with ERA was $63 \%$ but a decrease was seen in IL23p19 mRNA expression for in HLAB27 negative patients $(-3.8 \%)$ with the addition of TM. When HLAB27 positive patients not oh TNF inhibitors were compared to those on treatment, the median percentage increase between LPS and LPS + TM treated MDMs was significantly modulated [78.7\% vs $10.1 \%$, p-0.049]. mRNA expression for other pro-inflammatory cytokines including TNF, IL1 and IL6, in addition to markers of the UPR (XBP1, CHOP and BiP) demonstrated no significant differences between patients and healthy controls.

Conclusions: Expression of IL23p19 mRNA in MDMs is significantly enhanced in HLAB27 positive patients with ERA. In this group, the induction of the UPR appears to further enhance IL23p19 expression but this effect is modulated by treatment with TNF inhibitors. These results suggest a potential role for IL23 and the UPR in the pathogenesis of ERA, particularly in those who are HLAB27 positive and may have implications for treatment stratification, indicating a subgroup of patients who may respond to IL23 blockade.

Disclosure of Interest: None declared

DOI: 10.1136/annrheumdis-2017-eular.5004

\section{OP0057 FLUORESCENCE OPTICAL IMAGING IN JUVENILE IDIOPATHIC POLYARTICULAR DISEASE BEFORE AND DURING ANTIRHEUMATIC TREATMENT}

A. Klein ${ }^{1}$, G.-W. Just ${ }^{1}$, S.G. Werner ${ }^{2}$, I. Becker ${ }^{3}$, P. Oommen ${ }^{4}$, K. Minden ${ }^{5}$, H.-E. Langer ${ }^{2}$, G. Horneff ${ }^{1} .{ }^{1}$ Asklepios, Sankt Augustin; ${ }^{2}$ RHIO, Duesseldorf; ${ }^{3}$ University Cologne, Cologne; ${ }^{4}$ University, Duesseldorf; ${ }^{5}$ Charite, Berlin, Germany

Background: Valid detection of inflamed joints is essential for correct classification, therapeutic decisions and assessment of treatment efficacy in juvenile idiopathic arthritis (JIA)

Objectives: Fluorescence optical imaging (FOI) enables visualization of inflammation in arthritis of finger and hand joints and might be used in JIA.

Methods: A 24-week observational study in polyarticular JIA patients newly starting treatment with either methotrexate or a biologic was performed. Patients were evaluated clinically, by ultrasound B-mode, power-Doppler and FOI at baseline, week 12 and 24

Results: Of 37 patients enrolled, 24 patients started MTX and 13 a biologic for the first time (Etanercept $n=11$, Adalimumab and Tocilizumab one 1). Composite measures, mean JADAS 10 decreased significantly from 17.7 at baseline to 12.2 and 7.2 at week 12 and 24 respectively and JIA-ACR 30/50/70/100 response rates at week 24 were $85 \% / 73 \% / 50 \% / 27 \%$. In total 1110 joints were examined clinically, 990 by US/PD and 990 by FOI. At baseline/week12/week $2423.6 \% / 16.4 \% / 9.0 \%$ joints on hand and fingers were clinically active joints, by US $19.4 \% / 16.1 \% / 11.5 \%$ joints showed effusions, $18.8 \% / 12.7 \%$ and $9.6 \%$ showed synovial thickening and by PD $6.9 \% / 1.8 \% / 5 \%$ joints showed hyperperfusion. Any sign of arthritis was detected by US/PD in $24.5 \% / 19.2 \%$ and $17 \%$. By FOI at $38.7 \% / 29.2 \% / 27.6 \%$ showed a signal enhancement in at least one phase. Summarizing all 3 points

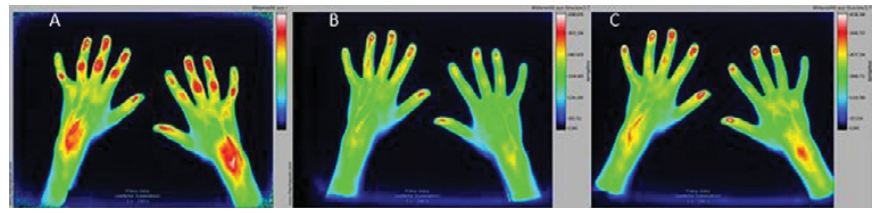

Figure 1. FOI composite images of a 14 year old patient with polyarticular JIA at start of etanercept treatment (left), after 3 (middle) and 6 months (right).

Abstract OP0057 - Table 1. Numbers of joints of a total of 2970 evaluated joints at 3 different points of time and comparison of number of joints with increased FOI signal and detection by US according to detection by clinical examination. Data on US examination were available for 2550 joints in total

\begin{tabular}{|c|c|c|c|c|c|c|c|c|c|}
\hline \multirow[b]{2}{*}{$\mathrm{n}(\%)$} & \multicolumn{5}{|c|}{$\mathrm{FOI}$} & \multicolumn{4}{|c|}{ US/PD } \\
\hline & $\begin{array}{l}\text { Any signal } \\
947\end{array}$ & $\begin{array}{l}\text { Composite image } \\
530\end{array}$ & $\begin{array}{c}\text { Phase } 1 \\
396\end{array}$ & $\begin{array}{c}\text { Phase } 2 \\
783\end{array}$ & $\begin{array}{c}\text { Phase } 3 \\
180\end{array}$ & $\begin{array}{l}\text { Any signal } \\
527\end{array}$ & $\begin{array}{l}\text { Effusion } \\
430\end{array}$ & $\begin{array}{c}\text { Synovitis } \\
375\end{array}$ & $\begin{array}{c}\text { Hyper-perfusion } \\
120\end{array}$ \\
\hline $\begin{array}{l}\text { Signal in clinically active joints } \\
(n=519)\end{array}$ & $\begin{array}{c}320 \\
(33.8 \% / 61.7 \%)^{*} r\end{array}$ & $\begin{array}{c}205 \\
(38.7 \% / 39.5 \%)\end{array}$ & $\begin{array}{c}161 \\
(40.7 \% / 31.0 \%)\end{array}$ & $\begin{array}{c}277 \\
(35.4 \% / 53.4 \%)\end{array}$ & $\begin{array}{c}81 \\
(45.0 \% / 15.6 \%)\end{array}$ & $\begin{array}{c}239 \\
(45.4 \% / 46.0 \%)\end{array}$ & $\begin{array}{c}211 \\
\operatorname{par}(49.1 \% / 40.7 \%)\end{array}$ & $\begin{array}{c}164 \\
164(43.7 \% / 31.6 \%)\end{array}$ & $\begin{array}{c}47 \\
(39.2 \% / 9.0 \%)\end{array}$ \\
\hline Signal in clinically inactive joints & $\begin{array}{c}627 \\
(66.2 \% / 25.6 \%)\end{array}$ & $\begin{array}{c}325 \\
(61.3 \% / 13.3 \%)\end{array}$ & $\begin{array}{c}235 \\
(59.3 \% / 9.6 \% 9)\end{array}$ & $\begin{array}{c}506 \\
(64.6 \% / 20.6 \%)\end{array}$ & $\begin{array}{c}99 \\
(55.0 \% / 4.0 \%)\end{array}$ & $\begin{array}{c}288(54.6 \% / 11.8 \%) \\
(54.6 \% / 11.8 \%)\end{array}$ & $\begin{array}{c}219 \\
(51.0 \% / 8.9 \%)\end{array}$ & $\begin{array}{c}211 \\
(56.2 \% / 8.6 \%)\end{array}$ & $\begin{array}{c}73 \\
(60.8 \% / 3.0 \%)\end{array}$ \\
\hline
\end{tabular}

${ }^{*}$ First percentage refers to columns, second to rows. 